\title{
A MODEL TWO-DIMENSIONAL POLYMERIZING FLUID FOR ADSORBED MONOLAYER OF CHAIN MOLECULES. INTEGRAL EQUATIONS AND MONTE CARLO SIMULATIONS
}

\author{
Yu. Duda ${ }^{1, \dagger}$, B. Millan-Malo ${ }^{2}$, O. Pizio ${ }^{1}$, D. Henderson ${ }^{3, *}$ \\ ${ }^{1}$ Instituto de Química de la UNAM, Coyoacán 04510, México D.F. \\ ${ }^{2}$ Instituto de Física de la UNAM, Coyoacán 04511, México D.F. \\ ${ }^{3}$ Department of Chemistry and Biochemistry, Brigham Young University, Provo, Utah 84602, USA \\ ${ }^{\dagger}$ Permanent address: Institute for Condensed Matter Physics, National Academy of Sciences of the Ukraine, Lviv-11, Ukraine
}

(Received August 1, 1997)

\begin{abstract}
The problem of adsorbed monolayer of chain molecules is considered. A polymerizing fluid of hard discs using the two-dimensional (2D) Wertheim-Ornstein-Zernike (WOZ) equation and the polymer Percus-Yevick (PPY) closure is studied. The formation of chains occurs due to the site-site associative interactions. Computer simulations for the model are performed. We have compared the structural properties from both methods to evaluate the possibility of application of the theory for more sophisticated models. It is shown that the PPY theory, supplemented by the ideal chain approximation, works quite well at low and intermediate densities for trimers and quadrimers At high densities, the quality of the theoretical predictions deteriorates. We find that the equation of state of Zhou et al. [J. Chem. Phys., 103, 2688 (1995)] supplemented by the effective chain length parameter of Jackson and Gubbins agrees well with our simulation data.
\end{abstract}

Key Words: monolayers, polymerizing fluids, density profiles, two-dimensional model.

PACS number(s): 61.20.Qg, 82.30.Nr, 61.25.Em, 61.25.Hq

\section{INTRODUCTION}

The statistical mechanical theory of chemically associating fluids (CAF) has developed rapidly during last decade. The theory that was pioneered by Wertheim $[1-4]$ is one of the most successful approaches in this area of chemical physics. This method has been successfully used to investigate chemical association phenomena (such as the formation of dimers and chains, for example) in the bulk fluids and mixtures, see eg. [5-11], as well as to study inhomogeneous CAFs [12-14]. The theory involves a multidensity Wertheim-Ornstein-Zernike (WOZ) integral equation and thermodynamic perturbation theory (TPT). The usual applications focus on three-dimensional (3D) systems. In particular, the structural and thermodynamic properties have been obtained for several models of associating fluids. The adequacy of the theoretical predictions has been thoroughly investigated and compared with computer simulations, see, e.g.[15-20] and references therein.

However, experimental studies of monolayer adsorption on well characterized surfaces indicate that, in many cases, the adsorbed molecules behave qualitatively similar to a two-dimensional (2D) fluid. The microscopic description of the monolayers adsorbed on homogeneous surfaces and their phase transitions has been the subject of many theoretical investigations. These include both computer simulations and integral equations. Most of this research has been concerned with simple 2D models, such as hard discs, Lennard-Jones discs, planar dumb- bells, see eg. [21-26]. However, the structure of a monolayer of chain molecules is more sophisticated than these simple fluids. This structure is of interest both for basic science and for several practical applications. One of the issues that is worth examining is the equation of state (EOS) of 2D chain fluids and 2D associating fluids. Very recently, Zhou et al. [27] have attempted to apply TPT for hard dumb-bells and hard chains made of discs. It has been shown that for a tangent hard-disc dimer fluid, the TPT is even more accurate than successful EOS of Boublik [24] from scaled particle theory. However, the absence of exact, i.e. computer simulations results, does not permit further progress in developing the equation of state for $2 \mathrm{D}$ polymerizing and 2D chain fluids.

Recently, we have initiated a study of 2D chemically associating fluids in our laboratory. In particular, the Monte Carlo simulation technique in the canonical NST ensemble has been applied for the 2D counterpart of the model of Cummings and Stell for chemical association [28]. A 2D model of polymerizing fused hard discs has been studied in ref. [29] also using the NST ensemble.

In the present work, our main concern is the solution of the WOZ equation for a 2D polymerizing fluid model, to study the results in the framework of computer simulations, and to present a comparison of the results from both approaches. A short-range nonassociative interaction between monomers is taken in the form of a hard disc potential. Each of the monomers contains two attractive sites such that the site-site association results in the formation of chain molecules. This model does not possess 
phase transitions, other than crystallization. Therefore, our focus is the structure of the monolayer rather than thermodynamic aspects related to phase transitions. It would be interesting to study such transitions in the framework of models with an attractive nonassociative interaction, such as the Lennard-Jones potential, for example.

In any case, the EOS of the model under study here is obtained. Our expectation is that the model will serve as a good reference to investigate ordering effects in a monolayer of short chains and chains of intermediate length on homogeneous and heterogeneous surfaces. Possible extensions of the model and theory are outlined in the concluding section.

\section{A MODEL AND PROCEDURE}

Let us consider a one-component fluid of polymerizing discs, a 2D counterpart of the model used by Wertheim for chain formation [4]. It is assumed that in an experiment the molecules that are able to form chains have been deposited onto a homogeneous structureless surface. Therefore, the mechanism of association does not involve surface structure. In spite of this idealization, it should be possible to initiate a systematic study of the monolayers made of chains via a chemical reaction of the Langmuir-Hinshelwood type mechanism.

The hard discs are chosen to be of the unit diameter, $d_{0}=1$. Each of discs has two attractive sites located inside its core. The sites are assumed distinguishable, they are denoted as $A$ and $B$. The interparticle pair potential is considered as a sum of the nonassociative contribution (spherically symmetric repulsion) and of strong attractive, finite range site--site interactions,

$$
U(12)=U_{n o n}\left(r_{12}\right)+U_{A B}(12)+U_{B A}(12),
$$

where 1 and 2 denote the positions $\mathbf{r}_{1}, \mathbf{r}_{2}$ and orientations $\Omega_{1}, \Omega_{2}$ of two particles (the orientation of particles are chosen in the laboratory frame using the position of site $A$ for the sake of concreteness), the distance between centers of particles is $r_{12}=\left|\mathbf{r}_{1}-\mathbf{r}_{2}\right|$. The nonassociative part of the total potential $U_{n o n}(r)$ is chosen in the form

$$
U_{\text {non }}(r)=\left\{\begin{array}{c}
\infty, \text { for } \quad r<L \\
D_{0}, \text { for } L<r<1, \quad\left(D_{0}>0\right) \\
0, \text { for } \quad r>1
\end{array}\right.
$$

the associative potentials $U_{A B}(12), U_{B A}(12)$ of site-site attraction are given as

$$
U_{G G^{\prime}}(x)=\left\{\begin{array}{cl}
-\left(\varepsilon_{a s}+D_{0}\right)\left[1-\delta_{G G^{\prime}}\right], & \text { for } x<\Delta, \\
0, & \text { for } x>\Delta
\end{array} \quad\left(\varepsilon_{a s}>0\right) .\right.
$$

Here $G$ denotes one of the sites, $A$ or $B$, and $G^{\prime}$ denotes its complementary site; $G G^{\prime}$ denotes $A B$ or $B A ; x$ is the distance between sites $A, B$. The sites having the same label do not interact, however. The bonding length parameter, $L$, is the distance between the centers of two associated particles. The parameter $\Delta$ is the range of site-site attractive square well that must guarantee only one bond per site for the formation of chain or ring-type structures, i.e. without branched configurations. To ensure this, a steric saturation condition,

$$
L<2 l_{d}+\Delta<L+(2-\sqrt{3}) l_{d}
$$

is imposed, where $l_{d}$ is the distance between the center of the hard sphere and the location of the site.

Similarly to the applications that have been developed for 3D systems, [5-8], we assume that the sites are independent of each other. Thus, formally the hard disc monomers are freely joined. However, the flexibility of a macromolecule is restricted due to the minimum value of the angle between two adjacent bonds, i.e. $2 \arcsin [1 / 2 L] \leq \Theta \leq \pi$. This restriction results from the impermeability of monomers next to the nearest neighbors. By changing the parameter of bond length, $L$, one can consider chains with different degree of flexibility, i.e. it is possible to form totally flexible chains $(L=1)$ up to almost completely rigid chains $(L \simeq 0.5)$. It is expected that the probability of the formation of ring-type structures would increase with increasing $L$.

The most important ingredient necessary in the following application of Wertheim's theory is the associative 'Mayer' function,

$$
F_{\text {as }}^{\text {or }}(1,2)=\exp \left[-\beta U_{\text {non }}\left(r_{12}\right)\right]\left\{\exp \left[-\beta U_{G G^{\prime}}(x)\right]-1\right\} \text {. }
$$

The theory considers $F_{a s}^{o r}(1,2)$ in its orientationally averaged form, $F_{a s}\left(r_{12}\right)$ [2], 


$$
\begin{aligned}
& F_{a s}\left(r_{12}\right)=\int d \Omega_{1} \int d \Omega_{2} \exp \left[-\beta U_{n o n}\left(r_{12}\right)\right]\left\{\exp \left[-\beta U_{G G^{\prime}}(x)\right]-1\right\} \\
& =\left\{\begin{array}{cc}
\exp \left(\beta \varepsilon_{a s}\right)\left(2 \Delta-2 l_{d}+r\right) \frac{\left(\Delta+2 l_{d}-r\right)^{2}}{24 l_{d}^{2} r}, & L<r<2 l_{d}+\Delta, \\
0, & \text { otherwise }
\end{array},\right.
\end{aligned}
$$

where $\int d \Omega$ denotes integration over orientation of a particle. However, the theory involves the orientational correlations via a special form of the Ornstein-Zernike $(\mathrm{OZ})$ equation and closure relations. Those are formulated for the partial correlation functions describing different bonding states of particles.

The associative model presented above yields the formation of chains and ring-type structures. The investigation of such a polydisperse system requires, a selfconsistent determination of the fractions of each species (chains and rings). This is a difficult task on its own and will be omitted for the sake of simplicity at this initial stage of our investigation of $2 \mathrm{D}$ models. We will neglect the possibility of ring formation by dropping the contribution of ring diagrams to the free energy, see Refs. $[4,5]$ for more detail. The ideal chain approximation (ICA) is used [5, 30]. The site-site association results in the formation of only chain molecules.

The WOZ matrix equation for the partial correlation functions reads [2]

$$
\mathbf{h}(r)=\mathbf{c}(r)+\int \mathbf{c}(s) \boldsymbol{\sigma} \mathbf{h}(|\mathbf{r}-\mathbf{s}|) d s
$$

where for the model in question the $4 \times 4$ matrix $\sigma$ is given by [5]

$$
\boldsymbol{\sigma}=\left(\begin{array}{cccc}
\sigma_{\Gamma} & \sigma_{B} & \sigma_{A} & \sigma_{0} \\
\sigma_{B} & 0 & \sigma_{0} & 0 \\
\sigma_{A} & \sigma_{0} & 0 & 0 \\
\sigma_{0} & 0 & 0 & 0
\end{array}\right)
$$

The density parameters $\sigma_{i j}(i, j=0,1,2,3)$ result from the classification of diagrams in the expansion of the oneparticle density $[2,4]$. These parameters are related to the partial densities $\rho_{\alpha}$ as follows,

$$
\begin{aligned}
& \sigma_{0}=\rho_{0}, \quad \sigma_{G}=\rho_{G}+\rho_{0}, \\
& \sigma_{\Gamma}=\rho_{0}+\rho_{G}+\rho_{G^{\prime}}+\rho_{\Gamma}=\rho,
\end{aligned}
$$

where $\rho$ is the total number density. The lower index $\alpha$ denotes the unbounded $(\alpha=0)$, the singly bonded $(\alpha=G)$, and the doubly bonded $(\alpha=\Gamma)$ states of a particle, respectively. The partial pair and direct correlation functions, $h_{i j}(r)$ and $c_{i j}(r)$ are the elements of the symmetric matrix $\mathbf{t}(=\mathbf{h}$ or $\mathbf{c})$

$$
\mathbf{t}=\left(\begin{array}{cccc}
t_{00} & t_{0 A} & t_{0 B} & t_{0 \Gamma} \\
t_{A 0} & t_{A A} & t_{A B} & t_{A \Gamma} \\
t_{B 0} & t_{B A} & t_{B B} & t_{B \Gamma} \\
t_{\Gamma 0} & t_{\Gamma A} & t_{\Gamma B} & t_{\Gamma \Gamma}
\end{array}\right)
$$

The PPY approximation is

$$
y_{i j}(r)=g_{i j}(r)-c_{i j}(r),
$$

where $g_{i j}(r)=h_{i j}(r)+\delta_{i 0} \delta_{j 0}$, and the functions $y_{i j}(r)$ are the analogues of the cavity correlation functions. These are related to the partial radial distribution functions $g_{i j}(r)$ via the definition [4],

$$
g_{i j}(r)=e^{-\beta U_{n o n}(r)} y_{i j}(r)+F_{a s} y_{00}(r) \delta_{i j}^{[1,2]},
$$

where we have used the notation $\delta_{i j}^{[1,2]}=\delta_{i 1} \delta_{j 2}+\delta_{i 2} \delta_{j 1}$.

In the ICA, the relation between the elements of the matrix $\boldsymbol{\sigma}$ is given as follows $[2,4]$

$$
\sigma_{\Gamma}=\frac{\sigma_{G} \sigma_{G^{\prime}}}{\sigma_{0}} ; \quad \frac{\sigma_{G}}{\sigma_{0}}=m=\frac{1}{2}+\sqrt{\frac{1}{4}+\rho I_{a s}},
$$

where $\eta_{\alpha}=\frac{1}{6} \pi \sigma_{\alpha}, \quad m$ is the mean number of beads per chain and

$$
I_{a s}=2 \pi \int_{0}^{\infty} d r F_{a s}(r) y_{00}(r) r .
$$

If the site-site potential is prescribed, equation (11) explicitly defines the mean chain length, $m$. We emphasize that such a parameter appears only in the framework of the ICA approximation. However, one can prescribe the mean chain length and choose the association energy $\beta \varepsilon_{a s}$ so as to provide a desirable $m$ at given density of particles. We choose this option to make a comparison with simulations, similarly to the previous studies of Chang and Sandler [5] in the case of 3D chains.

The total radial distribution function $g(r)$ is given in terms of the partial functions, $g_{i j}(r)$,

$$
\begin{aligned}
g(r) & =\frac{1}{\rho^{2}} \sum_{k n} \sigma_{0 k} g_{k n}(r) \sigma_{n 0} \\
& =1+h_{00}(r)+\frac{2}{m}\left[h_{0 G}(r)+h_{G 0}(r)\right]
\end{aligned}
$$




$$
+\frac{2}{m^{2}}\left[h_{G G}(r)+h_{G G^{\prime}}(r)\right]
$$

The results of WOZ-PPY approximation (complemented by the ICA) for the function $g(r)$ are discussed in comparison with the simulation data. However, first let us proceed with the discussion of the numerical algorithm that is necessary for the solution of the WOZ equations in two dimensions.

The method for solving the $2 \mathrm{D} \mathrm{OZ}$ equation has been presented in detail by Lado [31]. In contrast to the 3D case, which requires Fourier-transformation of the correlation functions, 2D models require Hankel-transforms of the correlation functions

$$
t_{\alpha \beta}(r)=\frac{1}{2 \pi} \int_{0}^{\infty} d k k J_{0}(k r) t_{\alpha \beta}(k),
$$

where both $r$ and $k$ are two-dimensional quantities, $J_{0}(x)$ is the zeroth-order Bessel function of the first kind and $t$ stands for $h$ or $c$ as appropriate. The Bessel functions has been calculated using a common routine [32]. The Hankel transforms has been obtained on a grid using the zeros of the Bessel function; the cutoff distance is chosen to be, $r=6$. Three hundred points on this interval has been taken. A similar number of points has been chosen for the inverse transformation.

\section{MONTE CARLO SIMULATIONS}

To evaluate the accuracy of the theoretical predictions we have performed NST simulations. The procedure is similar to that described in $[18,19]$. The unit cell in the $x y$ plane is a rectangle with length $l_{x}$ in $x$ direction and $l_{y}$ in $y$ direction. Periodic boundary conditions have been used in $y$ direction. We have restricted the system by impenetrable hard walls along $x$ axis at $x=0$ and $x=l_{x}$ This common method [18], has been used to obtain the pressure of the system using the contact theorem $\beta P=\rho(x=0)$, where $\rho(x)$ is the monomer density profile of the fluid near a hard wall. Care about the choice of the length of the simulation box in $x$ direction must be taken. Longer chains require a larger box because the bulk fluid properties are obtained from the central part of the box.

Initially, we choose $n$ hard discs and randomly insert them into the box ( $n$ is the number of chains desired). These discs are then grown into chains by the addition of beads using a 'growth and equilibration' algorithm [19]. Growth cycles, between attempts to move a randomly chosen molecule and attempts to add one bead to a randomly chosen molecule, are continued until all chains are completely grown to the desired number of participants. After the initial configuration has been generated in this manner, the system is evolved by moving a single, randomly chosen, molecule (chain). In the 'translational' move, the entire chain is first subjected to a randomly chosen displacement (uniform on the interval $[-a, a], a$ is an arbitrary constant chosen approximately between the monomer diameter and chain length) along each of the two coordinate axes. Following this, a slightly modified version of the continuum configurational bias algorithm [33] was used. At this stage, one of the chain end monomers is chosen at random, all other participants of the chain are deleted. Then a chain is regrown by adding beads sequentially. The parameters of all the moves was chosen so as to make each of the partial and the total acceptance ratio to be around $30-40$ per cent.

The system has been equilibrated, under different conditions, for at least $10^{4}$ simulation steps. A production run, during which the averages were obtained, consisted of at least $10^{5}$ steps. Two criteria were used to determine when equlibration was reached. These are the symmetry of the monomer density profile with respect to the restricting walls and the stability of the bulk density, i.e. in the. central part of the box.

The pair distribution functions were obtained from an analysis of the particle configurations in the bulk part of the simulation cell. We defined the bulk part of the cell from the shape of the monomer density profile and chose its length in $x$ direction to be slightly smaller than the region where the density profile is constant, apart from fluctuations. The desired density of the system was reached by the choice of the size of the unit cell and the number of monomers. We have verified carefully that the results do not depend on the cell dimensions.

\section{RESULTS AND DISCUSSION}

Let us proceed with the description of the results obtained. We discuss first the pair distribution function (pdf) of the particles, $g(r)$. They were obtained using the MC simulations and, theoretically, from eq. (13). The particles belong either to different chains or are participants of a single chain. In that latter case, the particles can be bonded via site-site association or they can be unbounded mutually if the next nearest neighbours are described (note that ring structures have been neglected using the IC approximation). Therefore, the function $h_{G G^{\prime}}(r)$ in the right hand side of eq. (13) contains two terms called the 'inter' and 'intra' chain contribution. The intermolecular contribution of $h_{G G^{\prime}}(r)$ is the function $h_{G G}(r)$. Thus, we also will discuss the intermolecular function $g^{\text {inter }}(r)$, obtained using eq. (13) but with $h_{G G^{\prime}}(r)$ substituted by $h_{G G}(r)$.

We begin our discussion with the case $m=3$, i.e. the 'chains' represent molecules that contain three monomers on average. The bonding length parameter, $L$, is taken close to unity, $L=0.9$ (figs. 1 and 2) such that the trimers are almost completely flexible. At low density, $\rho=0.227$ (fig. 1) the PPY approximation together with the ICA agrees quite well with the MC data. This agreement is excellent for the intermolecular function, $g^{\text {inter }}(r)$ (fig. 1b). At the higher density, $\rho=0.543$ (fig. 2) the intermolecular part also agrees well with simulations. However, the inaccuracies introduced by the PPY-IC 

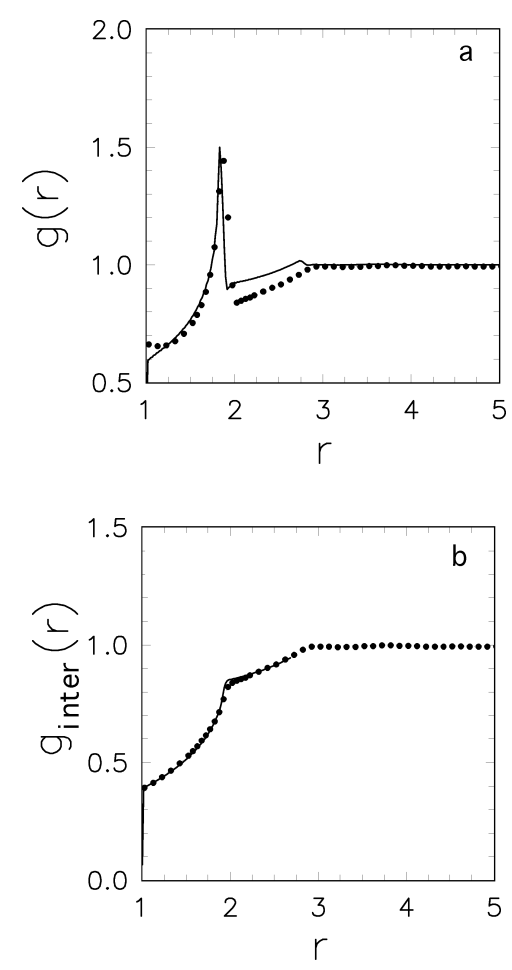

Fig. 1. The pair distribution function, $g(r)$ (part a) and the intermolecular pdfs, $g_{\text {inter }}(r)$ (part b), for a trimer fluid at the density $\rho=0.227$. The bonding length parameter is $L=0.9$. The solid line gives the results of the PPY closure with the ideal chain approximation. The points are the Monte Carlo simulation results.
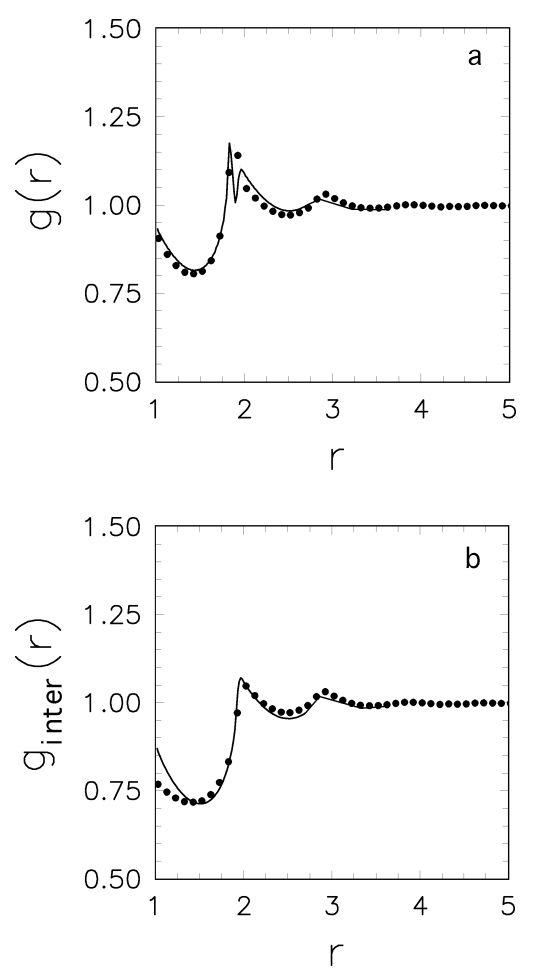

Fig. 2. The same as in fig. 1 but at the density 0.543 .
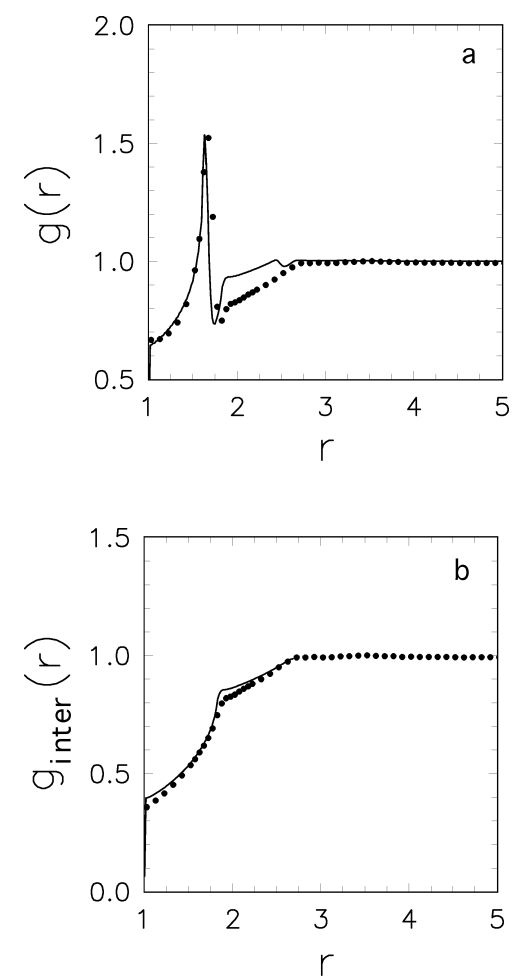

Fig. 3. The same as in fig. 1 but for a trimer fluid at the density 0.23 . The bonding length parameter is $L=0.8$.
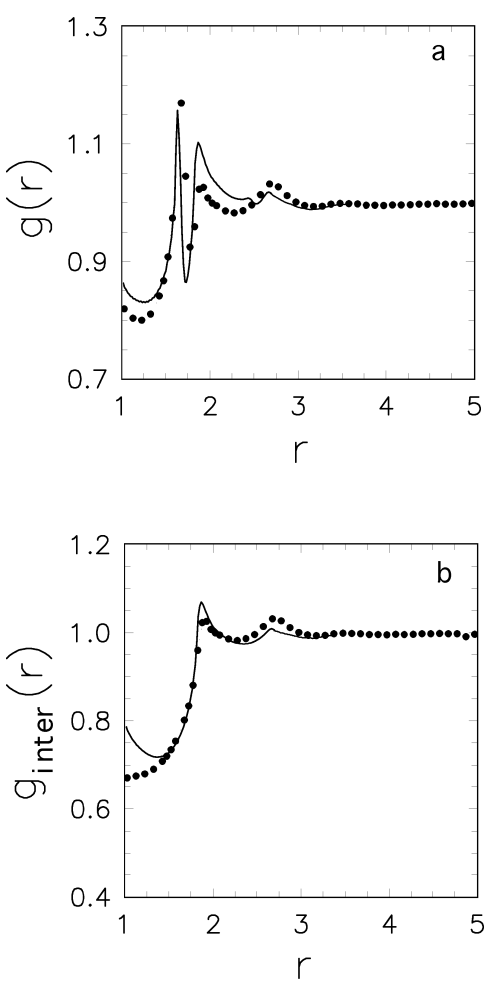

Fig. 4. The same as in fig. 3 but at the density 0.554 . 

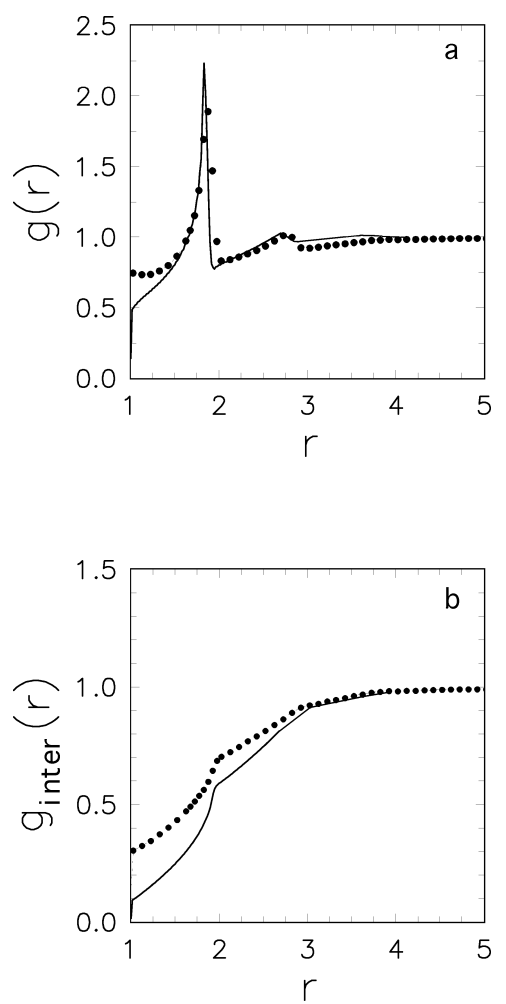

Fig. 5. The same as in fig. 1 but for quadrimer fluid at the density 0.211
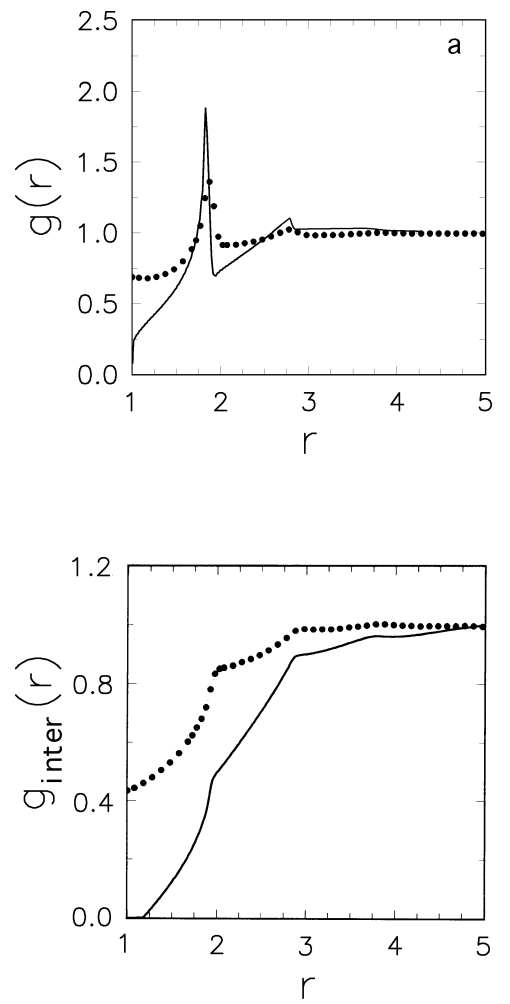

Fig. 6. The same as in fig. 5 but at the density 0.414 .
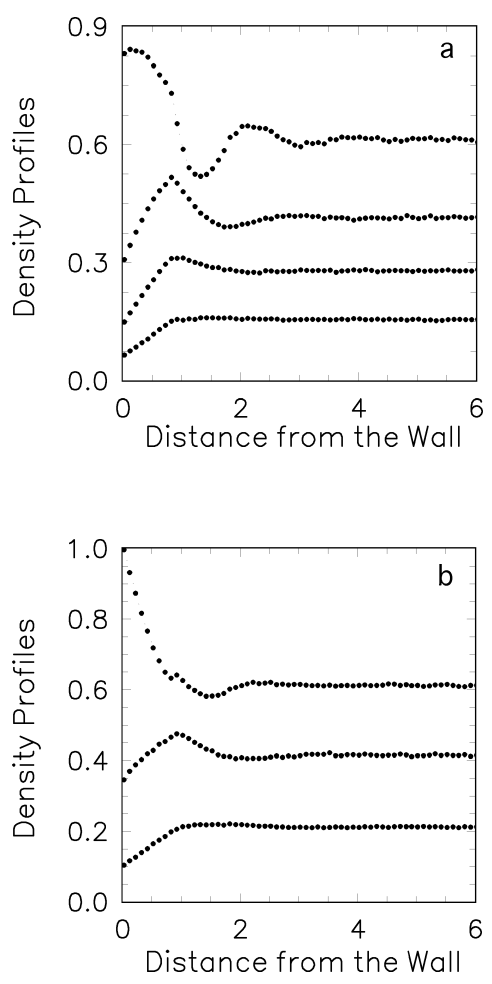

Fig. 7. The density profiles of monomers belonging to quadrimers at different fluid densities; these follow from the asymptotic values of the profiles. The bonding length parameter is $L=0.9$ (part a) and $L=0.8$ (part b).

approximation yield some discrepancies between the results from both approaches for $g(r)$ at $r=2 L$ (fig. 2a). In any case, for distances other than $r=1+L$ the pdf from the theory agrees well with simulation data.

Consider now the model with a slightly smaller bonding distance, $L=0.8$ (Figs. 3 and 4 ). In this case, the trimers that are formed due to association become less flexible in comparison with the previously discussed model, where $L=0.9$. At a low density, $\rho=0.23$ (fig. 3), the intermolecular part of the pdf is reproduced well by the PPY $+\mathrm{IC}$ approximation. The pdf $g(r)$ is less accurate, especially in the region $2 L<r<1+2 L$. However, this function remains qualitatively correct in comparison with the computer simulation data. At a high density, $\rho=0.554$ (fig. 4), the inaccuracies of both functions $g(r)$ and $g^{\text {inter }}(r)$, increase in magnitude. Qualitative agreement between the theory and simulations still remains, however.

In the case of a quadrimer fluid $(m=4)$, we also observe that the PPY $+\mathrm{IC}$ approximation works better at lower density, $\rho=0.211$ (fig. 5), than at a higher density, $\rho=0.414$ (fig. 6 ). At this higher density, $\rho=0.414$, only the trends of the behaviour of the pdf for quadrimer fluid remain similar in the theory and simulations.

We have obtained the density profiles of particles in the associating fluid near a hard line as a by-product of our simulations. These are shown for different densities in fig. 7. It is of interest to mention that for both models in question, $L=0.9$ and $L=0.8$, we observe depletion 
of the profiles at low densities whereas at high density the contact value of the profile is larger than at the bulk density. At low and intermediate densities, an absence of oscillations in the profiles of the chains is the characteristic feature of the profiles; only at high densities do the oscillations develop, similarly to the profile of a hard sphere fluid near a hard wall. In the high density region, chains with a larger degree of overlap between monomeric units usually exhibit a maximum value of the profile, not exactly at the surface line but at some small separation from it. These trends have been observed by Costa et al. [34] in computer simulations of hard alkane models near a hard wall. Our results for the profiles may serve as a background to study the behavior of chain molecules near extended line-type defects on the surface.

Finally, in Tables 1 and 2 we present some of the parameters of simulations together with our calculations of the values of the compressibility factor of the chains, $Z_{c h}$, $Z=\beta P m / \rho, m$ is the number of particles in the chain. We present the values for $Z$ which follow from our simulations as well as from the theory proposed recently by Zhou et al. [27] for 2D systems. In our computer simulations, we obtain the pressure from the contact value of the density profile of chains; the chain density has been evaluated in the bulk part of the simulation cell. In these theoretical approaches, which focus on the description of chains made of overlapping spheres, it is important to use the parameter of effective chain length, $m_{e f}$. In this work on $2 \mathrm{D}$ systems we apply the equation of state proposed by Zhou et al. [27] with two different choices of the effective chain length. Namely, we use the recipe pro- posed in [27] as well as the recipe of Jackson and Gubbins [35]. Those are denoted by $m_{e f}^{Z h}$ and $m_{e f}^{J}$, respectively. It appears that for the $2 \mathrm{D}$ systems in question with a restriction to the trimer and quadrimer cases, the theory based on the choice of Jackson and Gubbins works better than does $m_{e f}^{Z h}$. Longer chains must be studied separately. However, the agreement between computer simulation data for short chains with two different length parameters is quite good.

\section{CONCLUSIONS}

We have presented the solution of Wertheim's Ornstein-Zernike equation for polymerizing fused hard discs supplemented by the ideal chain approximation. The results for the pair distribution functions are compared with Monte Carlo simulation data for fluid of trimers and quadrimers. It is shown that the theory agrees well with simulation data in the region of low densities, especially that the intermolecular part of the pdf agrees very well with the simulations. At high densities the agreement deteriorates. Further, we conclude that for a better description of the structure a more sophisticated closure (than the PPY) must be applied, in conjunction with the ideal chain approximation. If the ideal chain approximation is not used, then for the moment there is no means to determine the average chain length. Some modification of the ideal chain approximation is required. We expect to investigate these issues in a separate work using extensive simulation data.

\begin{tabular}{c||ccc|ccc}
\hline & $L=0.8$ & & \multicolumn{3}{|c}{$L=0.9$} \\
\hline \hline$\rho_{c h}^{\text {bulk }}\left(10^{-5}\right)$ & 767 & 1413 & 1847 & 757 & 1380 & 1810 \\
$N_{c h}^{M C}$ & 66 & 40 & 54 & 65 & 40 & 53 \\
$Z_{c h}^{M C}$ & 1.701 & 2.696 & 3.899 & 1.771 & 2.884 & 4.475 \\
$Z_{c h}^{1}$ & 1.538 & 2.40 & 3.40 & 1.605 & 2.58 & 3.79 \\
$m_{e f}^{Z h}$ & 1.703 & & & 2.011 & & \\
$Z_{c h}^{2}$ & 1.651 & 2.742 & 4.064 & 1.71 & 2.901 & 4.43 \\
$m_{e f}^{J}$ & 2.6 & & & 2.8 & & \\
$<L_{e e}>$ & 1.484 & 1.487 & 1.491 & 1.625 & 1.618 & 1.612 \\
& & & & & & \\
\hline
\end{tabular}

Table 1. The MC simulation results for polymerizing disks: the density of the trimers, $m=3$, in the bulk region of the cell $\rho_{c h}^{b u l k}$, the number of chains in the simulation cell $N_{c h}^{M C}$, the compressibility factors from the simulations and from the theory with two choices of the effective chain length parameter. The average end to end distance is given in the last line of the table. 


\begin{tabular}{|c|c|c|c|c|c|c|c|c|c|c|}
\hline & \multicolumn{3}{|c|}{$L=0.51$} & \multicolumn{4}{|c|}{$L=0.8$} & \multicolumn{3}{|c|}{$L=0.9$} \\
\hline$\rho_{c h}^{b u l k}\left(10^{-5}\right)$ & 527 & 1040 & 1557 & 388 & 699 & 1041 & 1541 & 530 & 1037 & 1533 \\
\hline$N_{c h}^{M C}$ & 45 & 30 & 45 & 30 & 45 & 30 & 45 & 45 & 30 & 45 \\
\hline$Z_{c h}^{M C}$ & 1.66 & 2.135 & 3.052 & 1.61 & 2.074 & 2.885 & 5.331 & 1.859 & 3.262 & 6.569 \\
\hline$Z_{c h}^{1}$ & 1.32 & 1.81 & 2.58 & 1.40 & 1.90 & 2.76 & 5.29 & 1.61 & 2.79 & 5.34 \\
\hline$m_{e f}^{Z h}$ & & 1.43 & & & 2.46 & & & 2.53 & & \\
\hline$Z_{c h}^{2}$ & 1.41 & 2.05 & 3.16 & 1.45 & 2.00 & 2.90 & 5.65 & 1.75 & 3.26 & 6.66 \\
\hline$m_{e f}^{J}$ & & 2.53 & & & 3.4 & & & 3.7 & & \\
\hline$<L_{e e}>$ & 1.594 & 1.592 & 1.589 & 2.081 & 2.070 & 2.058 & 2.045 & 2.338 & 2.203 & 2.168 \\
\hline
\end{tabular}

Table 2. The MC simulation results for polymerizing disks yielding a quadrimer fluid, $m=4$. The other quantities are the same as in table 1.

On the other hand, we have shown that the equation of state of Zhou et al. [27] yields a compressibility factor that agrees well with simulations for both the trimer and quadrimer fluid. Future applications of this equation will become possible after performing simulations for longer chains in the framework of $2 \mathrm{D}$ model. It is of interest to extend this theoretical procedure for fluids with attractive interactions; these would yield two-dimensional phase transitions. However, an integral equation method may not be the most appropriate tool for this purpose. A density functional theory should be developed. This problem is now under study in our laboratory.

\section{ACKNOWLEDGMENTS}

This work has been supported in part by Silicon Graphics - Cray Research of Mexico under its University Research and Development Grant Program, by the DGAPA of UNAM under Research Project IN 20779, by the National Science Foundation (Grants No CTS94023584 and CHE96-01971) and the Petroleum Research Fund of the American Chemical Society (Grant No ACSPRF 31573-AC9). We are grateful to Profs. Yu. Kalyuzhnyi and S. Sokołowski for very helpful comments regarding this work. Interesting discussions with Prof. Yu. Holovatch are acknowledged with pleasure.
[1] M. S. Wertheim, J. Stat. Phys. 35, 17; 35 (1984).

[2] M. S. Wertheim, J. Stat. Phys. 42, 459; 477 (1986).

[3] M. S. Wertheim, J. Chem. Phys. 85, 2929 (1986).

[4] M. S. Wertheim, J. Chem. Phys. 87, 7323 (1987).

[5] J. Chang, S.I. Sandler, J. Chem. Phys. 102, 437 (1995).

[6] J. Chang, S.I. Sandler, J. Chem. Phys. 103, 3196 (1995).

[7] Yu. Kalyuzhnyi, C. T. Lin, G. Stell, J. Chem. Phys. 107, in press (1997).

[8] Yu. Kalyuzhnyi, P.T. Cummings, J. Chem. Phys. 105, 2011 (1996).

[9] A. Kovalenko, O. Pizio, D. Henderson, J. Phys. Chem. 101, 3571 (1997).

[10] Yu. V. Kalyuzhnyi, G. Stell, M.L. Llano-Restrepo, W. G. Chapman, M.F. Holovko, J. Chem. Phys. 101, 7939 (1994).

[11] Yu. V. Kalyuzhnyi, Condensed Matter Phys. No. 10, 51
(1997).

[12] D. Henderson, O. Pizio, S. Sokołowski, A. Trokhymchuk, J. Phys. Studies 1, 55 (1996).

[13] A. Trokhymchuk, O. Pizio, D. Henderson, S. Sokołowski, J. Phys. Chem. 100, 5941 (1996).

[14] A. Trokhymchuk, O. Pizio, M.F. Holovko, S. Sokołowski, J. Chem. Phys. 106, 200 (1997).

[15] G. Jackson, W.G. Chapman, K.E. Gubbins, Mol. Phys. 65, 1 (1988).

[16] J.K. Johnson, E.A. Muller, K.E. Gubbins, J. Phys. Chem. 98, 6413 (1994).

[17] C. Bokis, M. Donohue, C. Hall, J. Phys. Chem. 96, 11004 (1992).

[18] A. Yethiraj, C.K. Hall, Mol. Phys. 73, 503 (1991).

[19] A. Yethiraj, J. Chem. Phys. 101, 9104 (1994).

[20] N. A. Busch, M. S. Wertheim, M. L. Yarmush, J. Chem. 
Phys. 104,3962 (1996).

[21] E. Helfand, H. L. Frisch, J. L. Lebowitz, J. Chem. Phys. 34, 1031 (1961).

[22] W. A. Steele, J. Chem. Phys. 65, 5256 (1976).

[23] D. Henderson, Mol. Phys. 34, 301 (1977).

[24] T. Boublik, Mol. Phys. 63, 685 (1988).

[25] E. D. Glandt, D. D. Fitts, J. Chem. Phys. 66, 4503 (1977).

[26] K. Bagchi, H. C. Andersen, W. Swope, Phys. Rev. E 53, 3974 (1996).

[27] Y. Zhou, C. K. Hall, and G. Stell, J. Chem. Phys. 103, 2688 (1995).

[28] D. Henderson, A. Huerta, O. Pizio, A. Trokhymchuk, Mol. Phys. 90, 571 (1997).
[29] Yu. Duda, A. Trokhymchuk, S. Sokołowski, O. Pizio, J. Coll. Interface Sci. 194, 68 (1997).

[30] Yu. Duda, E. Vakarin, Yu. Kalyuzhnyi, M. Holovko, Physica A 245, 393 (1997).

[31] F. Lado, J. Comp. Phys. 8, 417 (1971).

[32] W. H. Press, S. A. Teukolsky, W. T. Vetterling, and B. P. Flannery, Numerical Recipes (Cambridge University Press, Port Chester, NY, 1986).

[33] J. J. de Pablo, M. Laso and U. W. Suter, J. Chem. Phys. 96, 2395 (1992).

[34] L. A. Costa, Y. Zhou, C. K. Hall, S. Carra, J. Chem. Phys. 102, 6212 (1995).

[35] G. Jackson, K. E. Gubbins, Pure Appl. Chem. 61, 1021 (1989).

\title{
ДВОВИМІРНА МОДЕЛЬ ФЛЮЇДУ З ПОЛІМЕРИЗАЩЕЮ ЧАСТИНОК ДЛЯ АДСОРБОВАНОГО МОНОШАРУ ЛАНЦЮЖКІВ МОЛЕКУЛ. ІНТЕГРАЛЬНІ РІВНЯННЯ І СИМУЛЯЩЯ МОНТЕ КАРЛО
}

\author{
Ю. Дуда ${ }^{1, \dagger}$, Б. Міллан-Мало ${ }^{2}$, О. Пізіо $^{1}$, Д. Гендерсон ${ }^{3}$ \\ ${ }^{1}$ Iнститут хемії УНАМ, Койоакан, 04510, Мехіко, Мексика \\ ${ }^{2}$ Iнститут фізики УНАМ, Койоакан, 04511, Мехіко, Мексика \\ ${ }^{3}$ Факультет хемії і біології Браям Янг, Прово, Юта, 84602, США \\ ${ }^{\dagger}$ Постійна адреса: Інститут фізики конденсованих систем НАН України, \\ вул. Свениічького, 1, Лъвів, 290011, Украӥна
}

\footnotetext{
У роботі розглянуто проблему опису моношару адсорбованих молекул. Вивчено властивості флюїду твердих дисків, кожен з яких має два притягальні центри, так що флюїд полімеризується. Застосовано рівняння Орнштайна-Церніке-Вертгайма у наближенні Перкуса-Свіка. Результати для парних функцій розподілу зіставлено з даними комп'ютерного експерименту і отримано добру згоду при малих густинах. При вищих густинах згода теорії ӥ експерименту погіршується. Обчислення тиску флюїду порівняно 3 результатами інших теорій.
} 